SAND-77-0242C

Unlimited Release

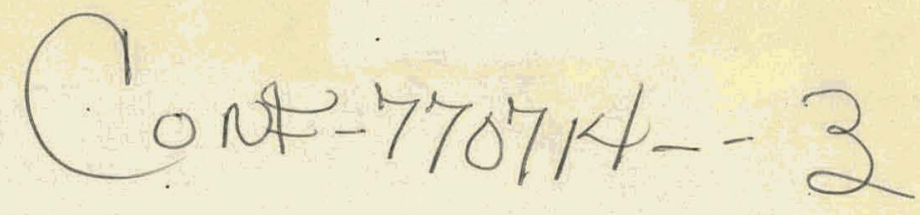

\title{
FIELD-ENHANCED REB DEPOSITION AND BREMSSTRAHLUNG PRODUCTION
}

J. A. Halbleib, Sr. and M. M. Widner

Prepared by Sandia Laboratories, Albuquerque, New Mexico 87115 and Livermore, California 94550 for the United States Atomic Energy Commission under Contract AT (29-1)-789

July 1977

Preprint

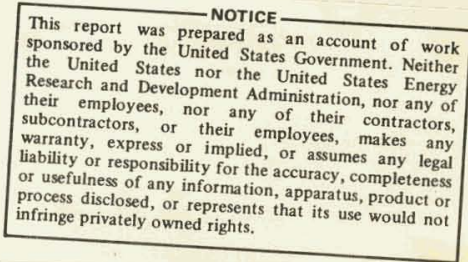




\section{DISCLAIMER}

This report was prepared as an account of work sponsored by an agency of the United States Government. Neither the United States Government nor any agency Thereof, nor any of their employees, makes any warranty, express or implied, or assumes any legal liability or responsibility for the accuracy, completeness, or usefulness of any information, apparatus, product, or process disclosed, or represents that its use would not infringe privately owned rights. Reference herein to any specific commercial product, process, or service by trade name, trademark, manufacturer, or otherwise does not necessarily constitute or imply its endorsement, recommendation, or favoring by the United States Government or any agency thereof. The views and opinions of authors expressed herein do not necessarily state or reflect those of the United States Government or any agency thereof. 


\section{DISCLAIMER}

Portions of this document may be illegible in electronic image products. Images are produced from the best available original document. 
Issued by Sandia Laboratories, operated for the United States Energy Research \& Development Administration by Sandia Corporation.

\section{NOTICE}

This report was prepared as an account of work sponsored by the United States Government. Neither the United States nor the United States Energy Research \& Development Administration, nor any of their employees, nor any of their con: tractors, subcontractors, or their employees, makes any warranty, express or implied, or assumes any legal liability or responsibility for the accuracy, completeness or usefulness of any information, apparatus, product or process disclosed, or represents that its use would not infringe privately owned rights. 


\section{Preprint}

SAND-77-0242C

FIELD-ENHANCED REB DEPOSITION

AND BREMSSTRAHLUNG PRODUCTION*

J. A. Halbleib, Sr. and M: M. Widner

Sandia Laboratories

Albuquerque, New Mexico 87115

July 1977

\section{ABSTRACT}

Recently developed models are employea to describe the interaction of \& high-current REB with planar gold foils in the presence of macroscopic electromagnetic fields. It îs show that, under certain conditions, azimuthal magnetic fields which either penetrate into the foil and/or exigt on the transmissinn side of the foil can significantly enhance the specific power deposited in the foil over that which would be deposited for diode fields alone. Similar field effects suggest methods for improving the external conversion efficiencies, softening. the spectra and focussing the source intensities of flash $x$-ray facilities. Finally, preliminary results are shown from a new trajectory-field model for self-consistent REB transport.

\footnotetext{
* This work was supported by the U. S. Energy Research and Development Administration.
} 
FIELD-ENHANCED REB DEPOSITION

AND BREMSSTRAHLUNG PRODUCTION

\section{Introduction}

The development and.maturation of high-intensity, pulsed relativistic electron beam ( $R E B$ ) technology has made possible the laboratory simulation of a great variety of nuclear and space radiation effects, the study of which could otherwise have been accomplished only through much more costly and time-consuming tests in the real environments. These diverse laboratory investigations range from observations of the response of complex systems in various radiation enviromnents to basic high-temperature, high-pressure materials research. $\mathrm{REB}$ simulation sources are generally employed in one of two ways, the direct electron mode or the flash $x$-ray mode. In either case the output of a particular facility is ultimately limited by the capacity of its energy storage unit. Given this constraint the utility of the source is likely to be determined by the efficiency with which the REB energy can be delivered to some chosen part of the experimental package in the direct electron mode, or by the efficiency with which it can be converted to bremsstrahlung in the flash $x$-ray mode. While substantial effort has been devoted to increasing the intensity of flash $\mathrm{x}$-ray sources for radiation effects simulation, efforts at improving specific energy deposition in the direct electron mode have, for the most part, been confined to electron-beam fusion research. 
In the next section we discuss a model developed in support of electron-beam fusion research, that employs macroscopic electromagnetic fields to predict enhanced specific energy deposition resulting from the interaction of a high-intensity $\mathrm{REB}$ and a metalic foil. It is further shown that an enhancement and/or spectral softening of the external bremsstrahlung is associated with this energy deposition. Finally, we discuss recent attempts to make this model self consistent, with specific application to the Alfvén problem.

\section{Enhanced Energy Deposition}

The model used for predicting the energy deposition resulting from the beara-foil interaction was a two-dimersional condensed-history ${ }^{1}$ Monte Carlo code employing relatively simple collisional electron transport. 2,3 Multiple elastic scattering of the primary electrons was described by the theory of Goudsmit and Saunderson, 4 and energy loss was accounted for in the continuous-slowing-down approximation ${ }^{5}$ (CSDA). No secondary production was allowed. Radiation loss by the primaries was included in the CSDA treatment, but the radiation itself was assumed to escape from the problem. The macroscopic electromagnetic fields were not self-consistent--instean a postulated self-magnetic field of the beam was simulated as an azimuthal magnetic field, $B_{\theta}$, which was a function only of the distance from the beam axis, $r$. The diode electric field, $\mathrm{E}_{z}$, was assumed to be a uniform axial field with a magnitude of $10^{6}$ volts $/ \mathrm{cm}$. The assumption of charge neutralization precludes the necessity for simulating space charge fields. This model is eimilar to that of Zinamon, et al. 6 
The configuration for the beam-foil interaction is shown schematically in Figure 1. An electron beam similar to that available from the HYDRA accelerator ${ }^{7}$ interacts with a planar gold anode foil of thickness $\Delta$. Histories are initiated at the left boundary of the anode foil. The source angular distribution was isotropic and source radii were sampled from the truncated Gaussian current-density profile.

$$
J(r)=\left\{\begin{array}{lc}
J_{0} \exp \left[-r^{2} /\left(1.44 x_{b}^{2}\right)\right] & r \leq r_{b} \\
0 & r>r_{b},
\end{array}\right.
$$

where $J_{0}$ is a constant and $r_{b}$ is the beam radius. When non-zero, $B_{\theta}(r)$ was always chosen to be consistent with $J(r)$. All calculations in this and the following section were performed for $r_{b}=0.2 \mathrm{~cm}$ and $J_{0}=10^{6} \mathrm{~A} / \mathrm{cm}^{2}$, leading to a total current, I, of $90.6 \mathrm{kA}$. The monenergetic source electrons had a kinetic energy of $800 \mathrm{keV}$.

In Figure 2 the specific power deposited in the focal region, $P_{S}(W / g)$, is plotted as a function of foil areal density, $p \Delta\left(\mathrm{g} / \mathrm{cm}^{2}\right)$, for three magnetic field conditions: (I) $B_{\theta}$ only in the diode region; (2) $B_{\theta}$ on both sides of the foil; and (3) $B_{\theta}$ on both sides of and within the foil. $P_{s}$ is defined as the power deposited within $r<r_{b}$, divided by the focal mass $M=\rho \Delta \pi r_{b}^{2}$. Since, for the cases of interest, significant target expansion occurs on the time scale of the REB pulse duration, expanded foils are considered with thicknesses $\Delta=0.01 \mathrm{~cm}$. (upper graph) and $\Delta=0.05 \mathrm{~cm}$ (lower graph). In both cases the foil density, $\rho$, is uniform. 
The results in Figure 2 may be interpreted by considering what happens as the areal density (or the mass density) decreases. Consider first the case of $\Delta=0.01 \mathrm{~cm}$. The areal density corresponding to normal density gold is about $0.19 \mathrm{~g} / \mathrm{cm}^{2}-$-larger areal densities correspond to higher mass densities and smaller areal densities, to small mass densities for the given thickness $\Delta$. For normal density gold it has been show ${ }^{8}$ that a magnetic field intensity somewhere between $10^{6}$ and $10^{7}$ gauss is required in order for field effects to compete with collisional effects. However, the maximum field resulting from the above gaussian profile is only $91 \mathrm{kG}$. Thus, at an areal density of $0.2 \mathrm{~g} / \mathrm{cm}^{2}$ the electron transport is collision dominated, and therefore, the presence of the magnetic field within the foil is of no consequence. Also, since the energy transmission coefficient with diode fields alone is only about 2.5 percent at this areal density, the existence of $B_{\theta}$ on the transmission side of the foil serves little purpose. The result is that all three curves coincide at large areal. densities.

As the areal density decreases from $0.2 \mathrm{~g} / \mathrm{cm}^{2}, P_{s}$ increases because the power deposited in the focal volume is decreasing more slowly than the focal mass. At an areal density just below the practical range, about $0.19 \mathrm{~g} / \mathrm{cm}^{2}$, the energy transmission with diode fields alone becomes large enough that $B_{\theta}$ on the transmission side leads to some enhancement via reinjection of a transmitted electron energy. Thus the curves with $\mathrm{B}_{\theta}$ on the transmission side begin to rise above the curve for diode fields alone at an areal density of about $0.15 \mathrm{~g} / \mathrm{cm}^{2}$.

As the areal density continues to decrease the energy transmission. 
For diode fields alone continues to increase, and with it the enhancement due to magnetically induced reentry. The lower curve peaks at an optimum o.rea.l density of about $0.1 \mathrm{~g} / \mathrm{cm}^{2}$ which can be understood in terms of the dependence of the axial deposition profile upon the areal density of the foil. The two upper curves continue to rise but remain coincident so long as transport within the foil is collision dominated.

Finally, at an areal density of about $0.05 \mathrm{~g} / \mathrm{cm}^{2}$ the density of the foil becomes so low that transport is no longer collision dominated when the magnetic field is present within the foil. Consequently, below this value, for $B_{\theta}$ everywhere, $P_{S}$ is enhanced with respect to $P_{s}$ for $B_{\theta}$ on both sides, but not within the foil. There are basically two ways in which $B_{\theta}$ within the foil can lead to enhancement. When the density becomes small the electrons begin to follow a cycloid-like path within the foil to a degree dependent upon the ratio $\Delta / r_{L}$, where $r_{L}$ is the Larmor radius. However, even at the minimum Larmor radius $(\sim 0.044 \mathrm{~cm})$ this ratio is only about 0.23 for $\Delta=0.01 \mathrm{~cm}$. Still, the maximum of the ratio does occur at the beam radius where the radial density of source particles is also very near maximum (maximum occurs at $0.17 \mathrm{~cm}$ ). The other way in which $B_{\theta}$ within the foil functions to enhance the focal deposition is by inhibiting lateral escape from the focal volume. Even though the aspect ratio, $\Delta / r_{b}$, is only $1 / 20$, in lie absence of $B_{\theta}$ more and more particles will Icak out of the lateral boundary of the focal volume as the density increases. Again, the fact that the radial density of source particles' is very near maximum at $r_{b}$ and the isotropic source distribution encourage this leakage. 
Consider now the case of $\Delta=0.05 \mathrm{~cm}$. Both $\Delta / \mathrm{r}_{\mathrm{L}}$ and $\Delta / \mathrm{r}_{\mathrm{b}}$ are now five times greater so thet, as Figure 2 shows, the enhancement in $\dot{P}_{s}$ for $B_{\theta}$, everymere over the case where $B_{\theta}$ is on both sides but not in the foil is always greater at any given areal density than it was for $\Delta=0.01 \mathrm{~cm}$. Finally, note that the mass density at which this enhancement becomes perceptible, where the two upper curves split, is roughly the same for the two values of $\Delta\left(\rho \Delta\right.$ of $.04 \mathrm{~g} / \mathrm{cm}^{2}$ at $\Delta=0.01 \mathrm{~cm}$ vs $\rho \Delta$ of $0.16 \mathrm{~g} / \mathrm{cm}^{2}$ at $\Delta=0.05 \mathrm{~cm}$ ). For the given magnetic field this is the effective mass density at which macroscopic field and collisional transport begin to compete---at higher densities transport is collision dominated and at lower densities field effects become more important.

We have also performed $P_{S}$ is $p \Delta$ calculations (not shown) for unexpanded foils $\left(\rho=19.3 \mathrm{~g} / \mathrm{cm}^{3}\right)$ in the range $p \Delta \geq 0.01 \mathrm{~g} / \mathrm{cm}^{2}$. Agreement within $\sim 10 \%$ with the curves in Figure 2 with $\Delta=0.01 \mathrm{~cm}$ is observed. Another feature of Figure 2 is that as $\rho \Delta \rightarrow 0$, with diode $B_{\theta}$ only, we approach the thin stopping power limit $P_{S}=I(A) \times p^{-1} \partial E / \partial x\left(V \mathrm{~cm}^{2} / g\right) \pi r_{b}^{2} \cos \theta=$ $1.68 \times 10^{12}$, where $\theta=$ average angle of incidence $=60^{\circ}$ for an isotropic distribution. Also seen in Figure 2 is the reduction in enhancement for the expanded target $\Delta=0.05 \mathrm{~cm}$ over that for $\Delta=0.01 \mathrm{~cm}$ for the case with $B_{\theta}$ in the diode and behind the foil. As. described above, this is a result of electrons exiting the focal volume radially due to their initial angular spread and to scattering.

The conditions for enhanced deposition relative to that obtained with dode fields alone are either (I) the presence of the B field to a depth of at least $r_{I}$ in the material (i.e., $r_{I}$ less than the resistive 
skin depth) and/or (2) the presence of $B_{\theta}$ on the transmission side of the foil. Implicit in the first condition is the requirement that the magnetic Reynolds number

$$
R_{m}=1: 26 \times 10^{-8} \sigma v_{\exp } r_{L}
$$

be much less than 1.0, where $\sigma$ is the material conductivity (mho/cm) and $v_{\text {exp }}$ is the expansion velocity $(\mathrm{cm} / \mathrm{sec})$. For those cases where the conductivity is so large that the skin depth is substantially less than $r_{L}$, the second condition can still provide the enhanced deposition. However, the second condition will only be effective provided that the areal density of the foil is significantly less than the practical range of the source electrons.

We should also note that for the cases of field penetration, while $P_{S}$ is large for thin foils $\left(\rho \Delta \sim 0.02 \mathrm{~g} / \mathrm{cm}^{2}\right)$, the fraction of the total power deposited is small $(\sim 12 \%)$ with the remaining power being carried primarily by transmitted electrons. The transmitted electrons are found to have a large velocity component $v^{\prime}$, parallel to $B_{\theta}$, and drift in the $+\mathrm{Z}$ direction (the beam direction) as a result of centrifugal forces. The $\nabla \mathrm{B}$ drift, on the other hand, requires large $\mathrm{v}_{\perp}$ and is in the $-\mathrm{Z}$ direction (opposite the beam direction) for $r<r_{b}$. The transmission loss for $r<r_{b}$ can thus be thought of as beam electrons scattering in the foil into a "loss cone" in velocity space $\left(v_{\|}>\sqrt{2} v_{\perp}\right)$. of course, the very existence of $B_{\theta}$ on the transmission side of the foil is predicated upon (1) transport of virtually the entire beam through the foil and (2) no current neutralization in the region behind the foil. 
The realization of these conditions involve the more general question of self consistency.

Estimates of deposition enhancement can be made by noting

$$
\widetilde{p}_{S}=\widetilde{I}\left(\rho^{-1} \partial E / \partial x\right) \rho x / \rho \widetilde{\Delta S},
$$

where $\widetilde{P}_{S}$ is the local specific power deposited, $\tilde{I}$ is the current incident on area $S, \rho x$ the areal density along the electron path (counting multiple passea), $\rho \widetilde{\Delta} s$ the mass of the material volume. In the limit as $x$ and $\widetilde{\Delta} \rightarrow 0$, this becomes

$$
\widetilde{\mathrm{P}}_{\mathrm{S}}=(\mathrm{x} / \widetilde{\Delta}) \mathrm{J} \rho^{-1} \partial \mathrm{E} / \partial \mathrm{x},
$$

and for straight, normal paths $(x=\widetilde{\Delta})$ we recover the thin stopping linit. The simplest physical interpretation of the deposition enhancement is that it results from an increased particle path length $(x>\widetilde{\Delta})$ within a the material, due to the gyromotion of the particle. A more quantitative definition is to note that $x / \widetilde{\Delta}=f v / u_{D}$, where $f$ is a factor of order. unity, $v$ is the electron speed, and $u_{D}$ the drift velocity component normal to the incremental area $S$ defined above. For the problem we are considering, we can show that the enhancement $v / u_{D} \propto I / I_{A}$, where $I_{A}=17000$ By amperes and where $u_{D}$ is given by centrifugal and $\nabla B$ drifts. Thus we would expect $P_{s} \propto I^{2}$, provided $P_{S} \ll P_{\text {max }}=V I / M$, where $V$ is the diode voltage.

To test this hypothesis we vary I holding other parameters fixed-$\mathrm{p} \Delta=0.01706 \mathrm{~g} / \mathrm{cm}^{2}, \Delta=0.01 \mathrm{~cm}, \mathrm{v}=8 \times 10^{+5}$ volts, truncated Gaussian beam and, current-density profile with $r_{b}=0.2 \mathrm{~cm}$, penetrating 
$B_{\theta}$ Pields, and isotropic incidence. This is shown in Figure 3. "We"find that for $I \sim 10^{5} \mathrm{~A}, \mathrm{P}_{\mathrm{S}} \propto \mathrm{I}^{2}$ and that $\mathrm{P}_{\mathrm{S}} \ll \mathrm{P}_{\max }$. In the range of $I \geqslant 5 \times 10^{5} \mathrm{~A}, \mathrm{P}_{\mathrm{S}} \propto \mathrm{I}$ since the beam is being depleted, i.e., $\mathrm{P}_{\mathrm{S}} \rightarrow \mathrm{P}_{\max }=$ $3.73 \times 10^{8} \mathrm{I}$. As the fraction of the beam deposited becomes large, however, the assumed $B_{\theta}$ on the back side of the foil is not consistent with the transmitted current.

\section{Enhanced Bremsstrahlung}

The enhancement of electron energy deposition in thin foils via self-induced and/or externally applied macroscopic electromagnetic fields should extend the radiation effects capabilities of a given REB facility when operated in the direct electron mode. In this section we investigate the obvious possibility that the enhanced energy deposition be accompanied by a commensurate increase in bremsstrahlung production. Furthermore, since the stronger enhancement appears to occur in the thinner foils, there is the possibility of obtaining flash $x-r a y$ sources with both higher-intensities and softer spectra for improved simulation of nuclear radiation effects.

The model of the previous section is actually part of a much more elaborate model for describing the hydrodynamic response of a target to time-dependent pulsed $\mathrm{REB}$ deposition in the presence of macroscopic electromagnetic fields. 2 Due to the complexity of the model us at whule the collisional transport was kept as simple as possible. The effects of energy loss straggling were not accounted for, and the production and transport of all secondaries were ignored. In this section we employ a model with more sophisticated collisional transport, 9 albeit without 
hydrodynamic capability, to predict the bremsstrahlung environment associated with the enhanced energy deposition.

All except one of the bremsstrahlung calculations were performed for unexpanded gold foils. Nevertheless, good agreement is obtained when the predictions of energy deposition are compared with those of Figure 2 as a function of areal density. For unexpanded foils field effects within the foil can be ignored because transport is collision dominated. 8 For the field condition of the lower and middle curves of Figure 2, the improved collisional model predicts slightly higher energy depositions in the unexpanded foils at small areal densities (which correspond to expanded foils in Figure 2). Indeed, at small areal densities and with the magnetic field on both sides of, but not in the foil, the improved collisional model predicts energy depositions which are in better agreement with the upper curves of Figure 2 than with the middle curves. In the unexpanded foils collisional scattering outside of the focal region is less effective, while in the expanded foils the magnetic field inhibits the lateral motion of the electrons within the foils. To verify this point the improved collisional model was used to calculate the energy deposition in a foil at an areal density of $0.01 \mathrm{~g} / \mathrm{cm}^{2}$, but expanded to a thickness of $0.01 \mathrm{~cm}$, with fields on both sides of, but not in the foil; the result was in excellent agreement with Figure 2 .

The results of the bremsstrahlung study for unexpanded foils are sumarized in Figure 4 as a function of the areal density of the foil. Figure $4 \mathrm{a}$ is a plot of the ratio of the radiation yicld* from these *The photon energy generated in the foil expressed as a percent of the source electron kinetic energy. 
beam-foil interactions to that of an infinite medium. With no fields at all, the ratio asymptotically approaches a value corresponding to saturated electron backscattering and no electron transmission. An axial electric field in the diode prevents electron backscatter so that the radiation yield asymptotically approaches the value for an infinite medium.

For radiation-effects applications, however, it is not the radiation yield which is of most practical importance, but rather the external bremsstrablung efficiency, $* *$ the bremsstrablung spectrum, and the spatial intensity of the bremsstrahlung source. Figure $4 \mathrm{~b}$ shows the ratio of the predicted $4 \pi$ external bremsstrahlung efficiency for two field conditions, to that for no macroscopic fields at all. At the larger foil thicknesses the penetrating magnetic field leads to little additional enhancement over that provided by the diode electric field alone, while at smaller foil thicknesses the magnetic field leads to substantially larger enhancement. While it is true that the zero-field efficiency is itself relatively small at these smaller foil thicknesses, the energy spectrum is softer. Because of the strong dependence of the bremsstrahlung cross section on electron energy, conventional flash x-ray sources tend to operate at high voltages and employ relatively. thick converters, both of which lead to relatively hard bremsstrahlung spectra. Thus, Figure $4 \mathrm{~b}$ suggests that tangential magnetic fields penetrating the converter-anodes should lead to more intense soft bremsstrahlung spectra 9 for certain radiation effects testing. The reason for the magnetic field enhancement at small foil thicknesses is demonstrated further in Figure 4c, **The photon energy extracted from the foil expressed as a percent of the source electron kinetic energy. 
wich shows the mean number of foil reentries per source electron. At small foil thicknesses the penetrating magnetic field inhibits the escape of a large number of transmitted electrons.

Another advantage provided by the magnetic field, that holds for large thicknesses as well, is its spatial concentration of the bremsitrahlung source. One measure of the degree of this concentration is the ratio of the energy deposited within the focal volume to the total energy deposited in the foil. This ratio is plotted in Figure 4d. For most foil thicknesses it is about 50 percent larger with the magnetic rield than without it.

\section{Self-Consistent Model}

In this section results of electron transport calculations with self-consistent magnetic fields and particle trajectories are presented. a This work is motivated by the predicted energy deposition enhancement $v / u_{b} \propto I / I_{A}$ which results from the non-self-consistent model as shown in Figure 3. For efficient deposition with the deposited power being a large fraction of the incident power, the beam must be substantially depleted and scattered. Therefore, the assumptions of a B field given by the incident beam is not valid. To properly account for depletion and scattering, self-consistency is required.

Self-consistent, párticle-field models which are quasi time dependent have been discussed in Ref. 3 and 10 and references contained therein, and have been used to study diode and beam transport calculations. Our Monte Carlo models cannot be used directly with this approach, however, since it is required that we follow a complete electron 
history until the electron either stops in a material or leaves the problem region. We have, therefore, adopted a field-trajectory approach similar to that described by Boers. 11 This technique consists of (1) assuming a B field, (2) calculating trajectories in the given B field, (3) computing current from the trajectories and a new B field from Ampere's law, and (4) iterating the procedure until some convergence criteria is satisfied. The end result is a steady-state solution. If collisions are included, the electrons move in these $B$ fields between scattering events as for the case of the non-self-consistent model. This approach has been used to investigate beams with $\nu / \gamma \leqslant 1.0$ with magnetic forces only. In particular we have concentrated on comparisons with time-dependent diode calculations previously reported by Poukey 12 to check the validity and accuracy of the numerical approach. An example of one such comparison is shown in Figure 5 which corresponds to a $v / \gamma=0.1$ beam with no collisions. The cylindrical beam is injected from the left boundary with uniform beam and current density. Figure 5a represents particle positions as reported by Poukey in Ref. 13, and Figure 5b shows sample trajectories from our model. Note the agreement .in the beam envelope, pinch location, and structure are excellent. In this calculation we used 101 particle trajectories and \& 13 (radial) by 40 (axial) uniform increment mesh. It was necessary to use 30 iterations starting with an initial guess of $B=0$ everywhere. Additional calculations have been performed for beams with $\nu / \gamma$ to 1.0 and work is in progress to extend this to larger values. Also, we have included material foils in situations similar to that shown in 
Figure 5, although these calculations become quite lengthy due to the statistical noise introduced by the Monte Carlo collisional model.

\section{Conclusions}

We have studied the effects of certain electromagnetic fields upon the interaction of a REB with gold foils. For the chosen azimuthal self-magnetic field, it was found that for this field to compete with collisional transport within the foil: (1) the foil must be expanded so as to reduce the collision frequency; and (2) the field must be able to penetrate the foil to a depth of the order of the Iarmor radius. Even if this latter condition cannot be met, the presence of an azimuthal self-magnetic field on the transmission side of the foil can still lead to a significant enhancement in the specific power deposition. In the case of unexpanded gold foils, it was found that macroscopic fields on either side of the foil can strongly effect the external efficiency, the energy spectrum and the spatial distribution of bremsstrahlung. These results suggest that externally applied and/or self-electromagnetic fields may be used to advance the technology of radiation effects simulation with $R E B^{\prime} s$.

Preliminary results from the application of a new self-consistent, particle-trajectory model to the Alfvén problem are encouraging. It is hoped that this model will lead to a more self-consistent description of important REB transport phenomena. 


\section{Acknowledgements}

It is a pleasure for the authors to acknowledge the many helpful discussions with James Poukey and Walt Vandevender, and the assistance provided by Mrs. Lessie Iee throughout the conduct of this research.

a 


\section{References}

1. M. J. Berger, Methods in Computational Physics (Academic Press, New York, 1963), Vol. I, p. 135.

2. M. M. Widner and J. A. Halbleib, Sr., Bull. Am. Phys. Soc. 20, 1272 (1975).

3. M. M. Hidner, J.W. Poukey, and J. A. Halbleib, Sr., Phys. Rev. Lett. 38, 548 (1977).

4. S. Goudsmit and J. I. Saunderson, Phys. Rev. 57, 24 (1940).

5. F. Rohrlick and B. C. Carlson, Phys: Rev. 93, 38 (1954).

6. Z. Zinamon, E. Nardi, and E. Peleg, Phys. Rev. Lett. 34, 1262 (1975).

7. T. H. Martin, IFEE Trans. Nucl. Sci. NS-20, 289 (1973). .

8. J. A. HaIbleib, Sr., IEEE Trans. Mucl. Sci. NS-21, 221 (1974).

9. J. A. Halbleib, Sr. and W. H. Vandevender, J. Appl. Phys., to be published.

10. J. W. Poukey, J. Vac. Sci. Technol. 12, 1214 (1975).

11. J. E. Boers, REEFER: A Digital Computer Program for the Simulation

- of High Energy Electron Tubes, SLA-73-0974, Sandia Laboratories, November 1976.

12. J. W. Poukey, A. J. Toepfer, and J. G. Kelly, Phys. Rev. Lett. 26, 1620 (1971). 


\section{Figure Captions}

1. Schematic of beam-foil configuration used in the calculation of energy deposition and bremsstrahlung production.

2. Specific power deposited in the focal region vs. foil areal density $\rho \Delta$. The symbols represent the following: ( $B_{\theta}$ fields

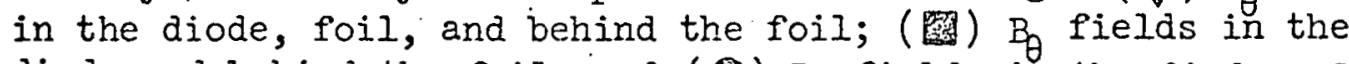
diode and behind the foil; and (2) B fields in the diode only.

3. $P_{S}$ vs. beam current for fixed beam voltage, beam radius, and foil thickness. The upper curve is the result if all the incident beam energy is deposited in the focal mass while the lower curve is the calculated deposition.

4. Bremsstrahlung results from beam-foil interaction as a function of foil areal density: (a) radiation yield as a fraction of the infinite medium radiation yield; (b) external bremsstrahlung efficiencies as a function of the zero field efficiency at the same foil thickness; (c) mean number of foil reentries per source electron; and (d) ratio of energy deposited in the focal volume to total energy deposited.

5. Self-consistent solutions of the Alfvén problem for a $v / y$ of 0.1 as obtained from the field-particle model (top) and the fieldtrajectory model (bottom). 
CONFIGURATION FOR MONTE CARLO CALCULATIONS

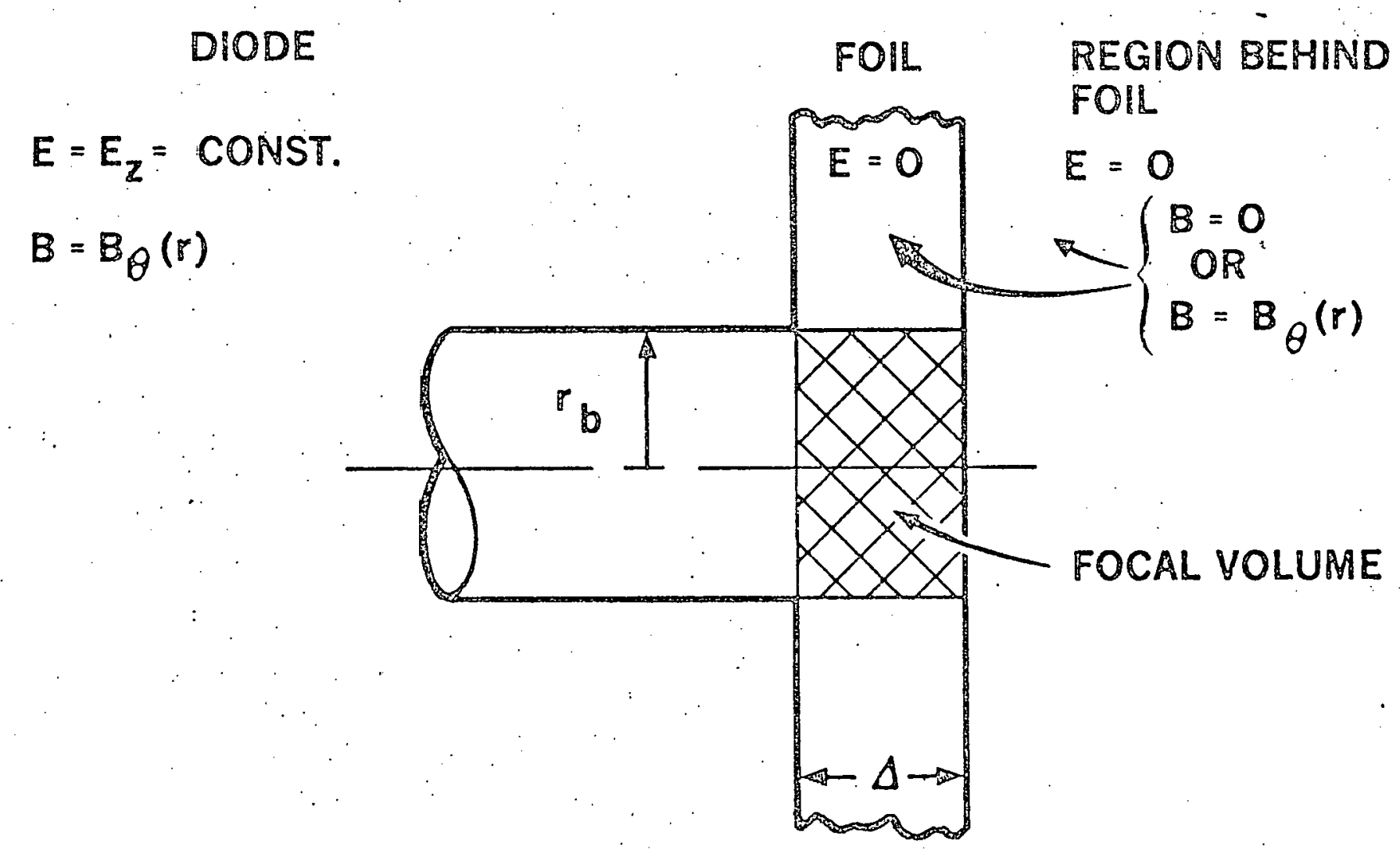

FIGURE 1 


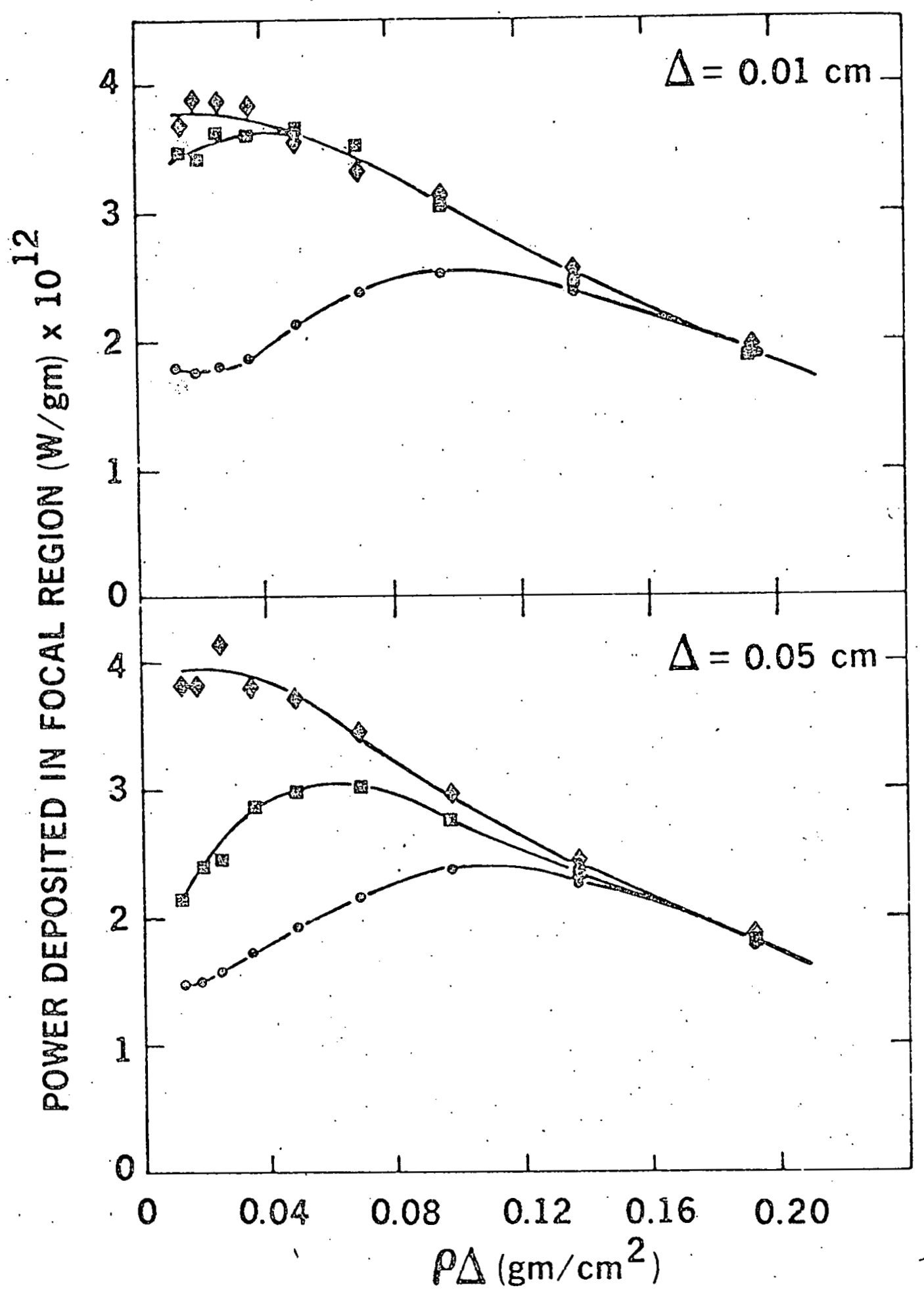

FIGURE 2 


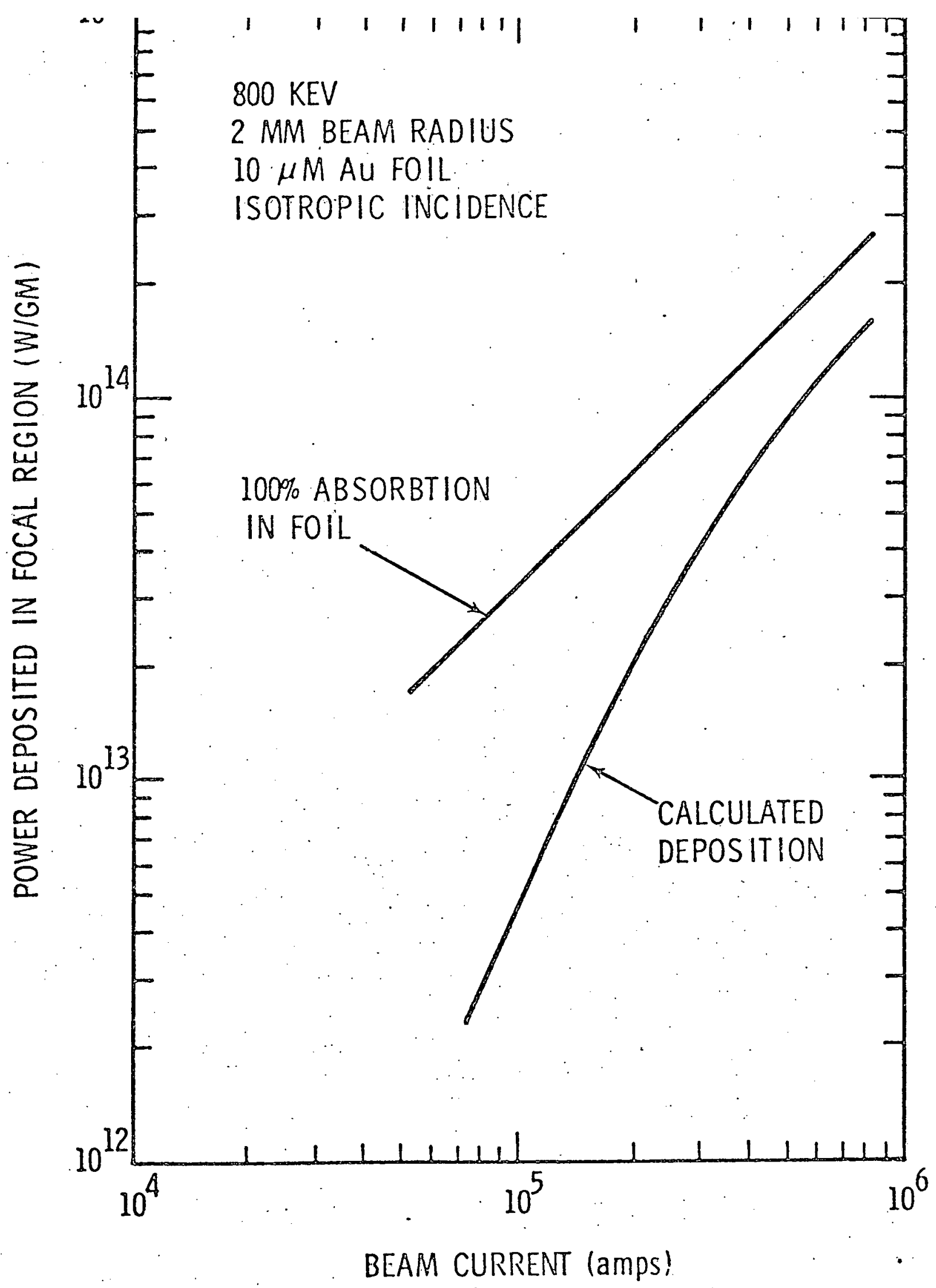

FIGURE 3 

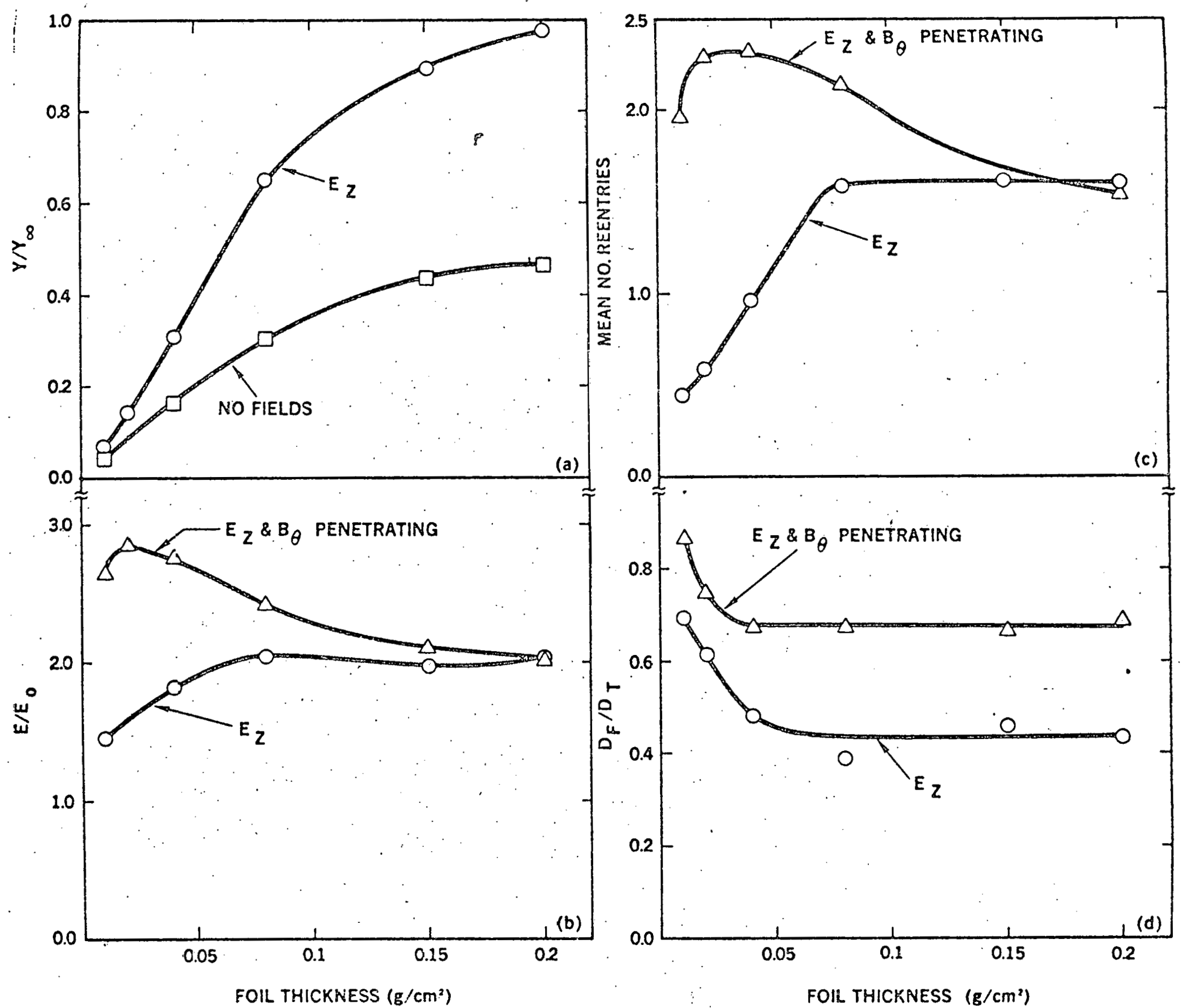

FIGURE $A$ 

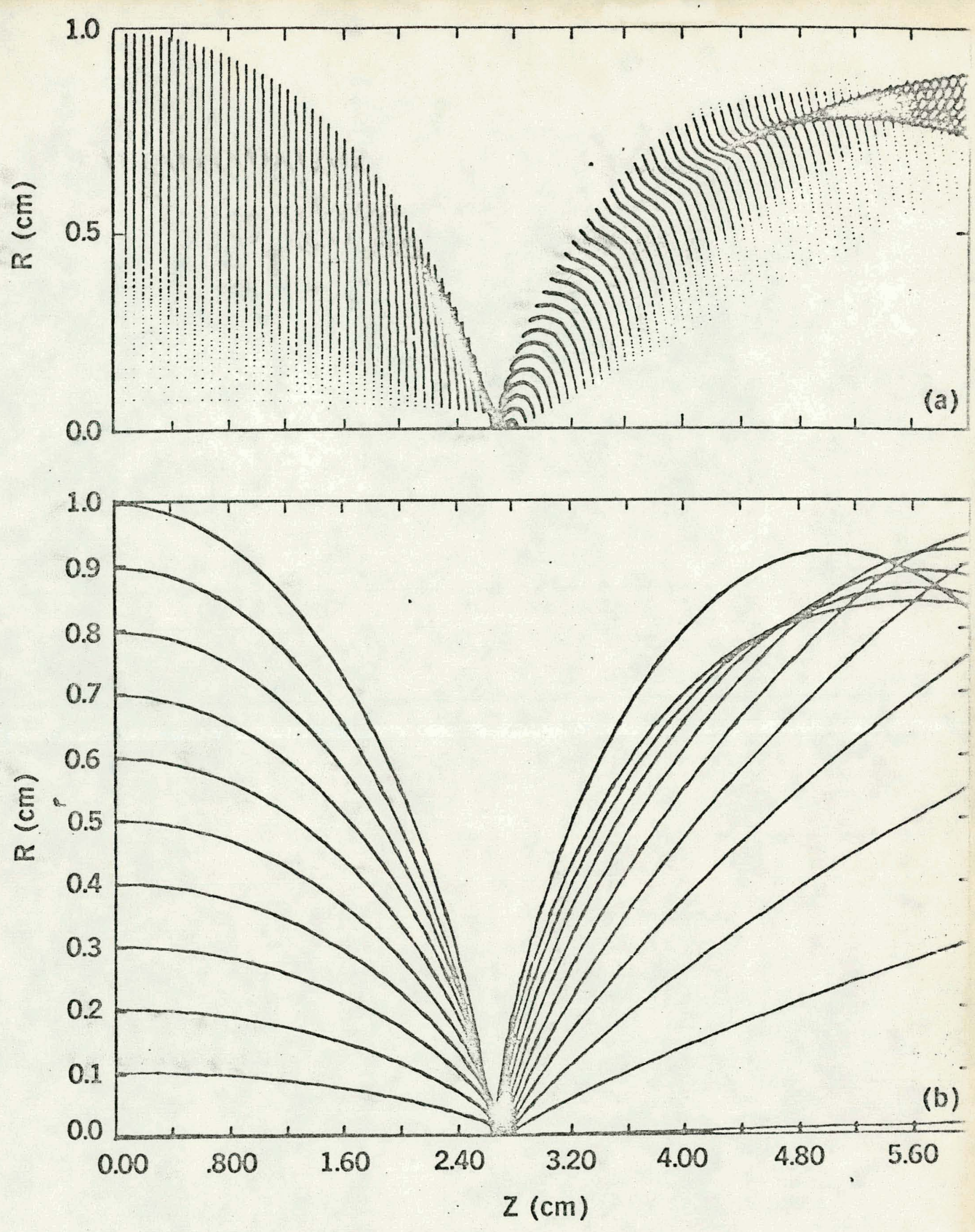

FIGURE 5 